Review Article

\title{
Multiple Applications of Different Exercise Modalities with Rodents
}

\author{
Denise Börzsei ${ }^{(D)}{ }^{1}$ Renáta Szabó $\left(\mathrm{D},{ }^{1,2}\right.$ Alexandra Hoffmann ${ }^{(D)}{ }^{1,3}$ Attila Harmath, ${ }^{4}$ \\ Judith Sebestyén, ${ }^{5}$ Jasmin Osman, ${ }^{1}$ Béla Juhász $\mathbb{D}^{6},{ }^{6}$ Dániel Priksz $\mathbb{D}^{1},{ }^{6}$ Csaba Varga $\mathbb{D}^{1,3}$ \\ and Anikó Pósa $\mathbb{D D}^{1,2}$ \\ ${ }^{1}$ Department of Physiology, Anatomy and Neuroscience, Faculty of Science and Informatics, University of Szeged, \\ Szeged 6726, Hungary \\ ${ }^{2}$ Department of Physiology, Anatomy and Neuroscience, Interdisciplinary Excellence Centre, University of Szeged, \\ Szeged 6726, Hungary \\ ${ }^{3}$ HR-Pharma Ltd., Szeged 6726, Hungary \\ ${ }^{4}$ KEL-FEDER PLC., Tompa 6422, Hungary \\ ${ }^{5}$ Department of Burns and Plastic Surgery, South-Pest Hospital Centre, National Institute for Infectology and Haematology, \\ Budapest 1097, Hungary \\ ${ }^{6}$ Department of Pharmacology and Pharmacotherapy, Faculty of Medicine, University of Debrecen, Debrecen 4032, Hungary
}

Correspondence should be addressed to Anikó Pósa; paniko@bio.u-szeged.hu

Received 1 July 2021; Revised 14 September 2021; Accepted 12 November 2021; Published 25 November 2021

Academic Editor: Tiago Fernandes

Copyright ( 2021 Denise Börzsei et al. This is an open access article distributed under the Creative Commons Attribution License, which permits unrestricted use, distribution, and reproduction in any medium, provided the original work is properly cited.

\begin{abstract}
A large proportion of chronic diseases can be derived from a sedentary lifestyle. Raising physical activity awareness is indispensable, as lack of exercise is the fourth most common cause of death worldwide. Animal models in different research fields serve as important tools in the study of acute or chronic noncommunicable disorders. With the help of animal-based exercise research, exercise-mediated complex antioxidant and inflammatory pathways can be explored, which knowledge can be transferred to human studies. Whereas sustained physical activity has an enormous number of beneficial effects on many organ systems, these animal models are easily applicable in several research areas. This review is aimed at providing an overall picture of scientific research studies using animal models with a focus on different training modalities. Without wishing to be exhaustive, the most commonly used forms of exercise are presented.
\end{abstract}

\section{Introduction}

Sport can be thought of as a therapeutic tool or a prevention strategy for different disorders. It is beginning to be learned that physical exercise exerts its effects via extensive molecular pathways by which it maintains and improves the quality of life. It is well known that regularly maintained training has several beneficial effects on overall health, from cells to whole organ systems [1]. Nonetheless, physical inactivity entails numerous health issues from systemic inflammation to hormonal dysfunctions which raises the risk of wideranging noncommunicable diseases, such as type II diabetes, metabolic syndrome, cardiovascular and neurodegenerative disorders, and even cancer [2-5]. Over and above, sedentary lifestyle-related redox disturbance further aggravates preexisting pathological processes [6]. The main purpose of training-related research studies has been targeted on the health benefits of exercise to be able to prevent and treat these conditions. Comprehending the underpinning systemic changes provoked by exercise helps us to develop more efficient treatment methods and prevention strategies against widespread diseases. With the help of animal models, it is possible to study the most complex effects of exercise at all levels of organization. The appropriate animal species and the duration, frequency, and intensity of the training should be chosen according to the purpose of the study 
[7]. The most common types of exercise used in animal experiments are voluntary wheel running, forced wheel running, treadmill running, swimming, and resistance training $[8,9]$. Aerobic exercise modalities are suitable for almost every noncommunicable disorder-related research area, while anaerobic training modalities are applicable in a much narrower field of research, including muscle formation studies [8-11]. In this review, we discuss the far-reaching benefits of physical exercise and its interpretation in different animal models. Our aim is to provide a comprehensive picture of the different exercise modalities used with rodents and their far-reaching effects on organ systems affecting the most researched noncommunicable diseases.

\section{Beneficial Effects of Exercise on Different Organ Systems}

2.1. Bones and Skeletal Muscle. Exercise has major effects on body composition. It is well established that physical activity improves bone properties such as bone quality or density. Consequently, it lowers the risk of osteoporosis. Osteoporosis is a condition characterized by low bone mass and bone fragility and mainly occurs among elderly people and postmenopausal women as a result of hormonal changes [12]. Exercise is considered to be the best nonpharmacologic approach in preventing osteoporosis; recent studies discussed that long-term exercise is able to increase bone strength and formation; therefore, it is effective in improving bone quality $[13,14]$.

Along with bones, exercise is able to increase muscle strength and improve balance and coordination. The most noticeable effect of long-term exercise, especially resistance training, is the increase of muscle mass. This process affects the basal metabolic rate and body composition in a favorable way. Besides, physical activity is proved to be promising in the regeneration and rejuvenation of muscle stem cells [15]. Exercise is also effective in age-related muscle atrophy, called sarcopenia. A recent work of White et al. supported the fact that long-term voluntary exercise can prevent sarcopenia in the hindlimb muscles in female and male rats as well [16]. Taken together, these results indicate that regular exercise has many beneficial effects on skeletal muscle function, regeneration, and bone quality at any age.

2.2. Metabolic Health. In the absence of exercise as a result of chronic positive energy balance, weight gain occurs. In this pathological condition, an increase in the number and size of adipocytes is observed, which leads to the disruption of leptin signaling and eventually to chronic inflammation [17]. On the contrary, exercise promotes metabolic health by decreasing body weight along with the amount of circulating lipids and the concentration of leptin and positively affects glucose tolerance and insulin sensitivity $[18,19]$. Studies have shown that regular exercise significantly improved glucose homeostasis in diabetic and prediabetic status [20]. Moreover, regular exercise is efficient enough to reduce plasma leptin and insulin levels in hormone deficiency as well; thus, it plays an important part in the improvement of pathophysiological changes in connection with metabolic syndrome [21, 22]. Furthermore, exercise is able to increase the expression of glucose transporter 4 and contributed to balanced glucose homeostasis and insulin sensitivity in rats [23]. Hence, regular exercise is able to reduce the risk for metabolic disorders and the resulting cardiovascular complications.

2.3. Cardiovascular System. Physical exercise has farreaching cardiovascular effects as well. Studies have shown that sustained physical activity lowers the individual's resting heart rate and blood pressure while it increases physiological cardiac hypertrophy [24, 25]. Exercise affects the cardiovascular system in different ways; it modulates numerous signaling pathways and improves oxygen delivery throughout the body via angiogenesis and vasodilation [26]. Nitrogen monoxide (NO) production in the endothelium rises significantly as a result of training and causes a well-known vasodilating phenomenon [27]. By enhancing nitrogen monoxide synthase activity, exercise has an undeniable role in the maintenance of normal blood pressure and in the treatment of hypertension [28]. Besides vasodilatation, NO has anti-inflammatory and platelet inhibitory effects as well, thereby contributing to the mitigation of atherosclerotic risk [29]. Additionally, exercise influences blood vessel morphology by extending the capillary network in the cardiovascular system. In a recent study of ours, we proved that a 12-week-long voluntary exercise was an effective therapeutic tool to improve cardiac function in aged rats; we clarified the exercise-moderated genetic modifications that contributed to the functional improvement of the heart [30]. Exercise can serve as a therapeutic tool after myocardial infarction (MI) as well; recent studies supported that after a postmyocardial injury, recreational exercise was able to improve cardiac health and antioxidant status $[31,32]$.

2.4. Nervous System. Numerous studies demonstrated the effects of physical activity on mental health, cognitive processes, and brain activity [33-35]. It is clarified that exercise affects several complex mechanisms including cerebral perfusion, neurogenesis, and synaptic plasticity [36, 37]. The findings of Kleemeyer et al. discussed that 6 months of exercise is associated with a hippocampal neuron density increase [38], while the work of Ruscheweyh et al. discovered a significant augmentation of the gray and white matter as a result of aerobic training [39]. Interestingly, encouraging results were obtained not only in young rats but also in older animals (19-month-old rodents). Scientists found that 1.5 months of voluntary exercise elevated gliogenesis by $20 \%$ and reverted age-related decline in neurogenesis by $50 \%$ [40]. Trophic factors such as the brain-derived trophic factor (BDNF) and vascular endothelial growth factor (VEGF) greatly support the cognition in young and older individuals as well. Several studies verified that running exercise at any age increased the expression of BDNF and VEGF in the hippocampus, which the phenomenon was correlated with the improvement of spatial learning and memory [41, 42]. In addition, exercise has been recommended as one of the best lifestyle interventions against neurodegenerative diseases (e.g., Alzheimer's disease, Parkinson's disease) 
[43]. It is well established that exercise has a crucial role in the protection of neurons, but recent results have also suggested that it is promising in the prevention of amyloid- $\beta$ and tau protein plaque formation [44, 45]. According to recent studies, swimming, voluntary wheel running, and treadmill exercise have also proven to treat neuropathic pain in a mouse model $[46,47]$. In addition, regular exercise is well known to trigger the release of serotonin and dopamine, which neurotransmitters help to overcome the symptoms of depression and anxiety [48].

\subsection{Antioxidant and Anti-Inflammatory Effects of Exercise.} A number of studies have shown that besides many favorable effects on different organ system functions (e.g., cardiovascular or metabolic), physical exercise is able to decrease proinflammatory markers and improve antioxidant status systemically [49-51]. Regular exercise mitigates reactive oxygen species-mediated cell damage by boosting antioxidant functions and reducing C-reactive protein, interleukin-6, and tumor necrosis factor-alpha (TNF- $\alpha$ ) levels [52, 53]. Furthermore, it is proved to reduce reactive oxygen species (ROS) production; therefore, it plays a key role in the maintenance of redox balance [54]. Regular training leads to the adaptation of the antioxidant capacity and protects the cells against adverse oxidative processes [55]. Sustained exercise has been demonstrated to be essential not only in the elimination of oxidative stress but also in the prevention of the abovementioned complex disorders, such as type II diabetes, metabolic syndrome, and cardiovascular and even neurodegenerative diseases [56]. Numerous animal studies also demonstrated the antioxidant effects of physical exercise by the enhancement of several enzymatic pathways including glutathione (GSH) and the heme oxygenase $(\mathrm{HO})$ system $[57,58]$. Szabo et al. supported that 12 weeks of sustained training is an efficient method to enhance the antioxidant GSH and nitrotyrosine-3 levels as well [59]. Moreover, in a hormone-depleted rat model, 6 weeks of physical exercise was proved to be a key process in the amelioration of antioxidant status by enhancing the $\mathrm{HO}$ enzyme system [31]. Besides GSH and HO, superoxide dismutase (SOD) is also considered to be a first-line defense participant against oxidative stress. According to animal studies, a significant elevation can be observed in the production of SOD as a result of recreational training $[60,61]$. Proinflammatory markers (e.g., myeloperoxidase (MPO), TNF- $\alpha$, and IL-6) are the main contributors of ROS generation and consequently oxidative stress. Exercise, however, has also been shown to be effective in the reduction of these wellknown inflammatory factors. Several studies showed that increased physical activity resulted in diminishing inflammatory processes $[49,62,63]$. It is clarified that 6-weeklong voluntary exercise is effective enough to reduce MPO activity and the level of TNF- $\alpha$ in hormonedepleted rats [64]. It can therefore be concluded that exercise represents a potent anti-inflammatory and antioxidant strategy in healthy individuals and under pathological conditions as well. It participates in reducing the risk of morbidity and mortality through its direct and positive impacts on human health.
2.6. Cancer Prevention. Studies provide numerous evidence that physical activity reduces the risk of at least a dozen cancer types, including breast, colon, prostate, or lung cancer [65]. In different chemical-induced or genetic tumor models, all of the training modalities mentioned in this review have been shown to be effective in reducing tumor growth or metastasis $[66,67]$. However, the underlying mechanisms of this wide-ranging protection are not yet totally clarified, but the possible mediators are inflammation-, antioxidant-, and immune cell-related [68]. During physical activity, a significant increase in muscle-derived myokines and intensified mobilization of immune cells can be observed in the plasma. While myokines have antiproliferative effects, immune cells can be the most powerful components in the fight against cancer $[69,70]$. Pedersen et al. found a marked decrease in tumor incidence and growth as a result of voluntary wheel running in 5 different tumor types. They clarified that natural killer cells have a predominant role in this type of control of tumor growth; with the induction of stimulatory cytokines, enhancement of NK cell-related activated receptors, and their intensified mobilization, they have a major role in the training-related control of tumor growth [71]. Moreira et al. revealed that even a short-term voluntary exercise decreased tumor growth and metastatic processes in a tumor-bearing rat model [72]. A promising observation was made by Hagar et al. as well that 8 weeks of training enhanced antitumor immune processes, thus suppressing tumor growth in mice [73]. Furthermore, aerobic exercise resulted in enhanced tumor cell apoptosis, decreased tumor weight, and diminished cell proliferation in a tumorbearing rat model compared to sedentary animals [74]. Physical activity has an unquestionable role in the primary prevention of cancerous processes; however, it is also extremely important in terms of health promotion after the diagnosis. With the help of exercise, aerobic capacity and muscle strength increase, while disease-free survival may extend [75].

\section{Characteristics of Animal Exercise Protocols}

Animal models are essential in basic research including every research field; thereby, choosing a well-designed exercise protocol for the appropriate experiments is fundamental. Before initiating any exercise study, the most important step is the proper selection of the animal model, as the objectives of exercise-related research studies may be different. For exercise training, rodents (rats and mice) are the most commonly used animals due to their many advantages. Rodents are the most affordable species for animal studies, thanks to their low breeding cost. They have high fertility and a relatively short gestational period with many offspring. Another advantage of using rodents is that they require comparatively small living space, and the experimental apparatus designed for mice or rats are also easily accessible [76]. Additionally, the capability of choosing genetically modified strains designed for specific diseases has also popularized these species in every research field. Despite the several advantages of rodents in animal research, few limitations are present in their application. In most animal 
studies, including exercise research, gender differences may interfere with the results and make it difficult to generalize data for both sexes, and although human and rodent genes are largely similar, the small but more important differences in the details (e.g., receptors) make rodents unsuitable for some research areas. Considering all aspects, after choosing the applicable animal model, we must consider the elementary factors of exercise. Physical activity can be characterized according to its intensity, duration, modality, and frequency $[7,77]$ (Figure 1).

Within the intensity of the training, we can distinguish between the low- and high-intensity physical activities; in terms of modality, we can differentiate between dynamic training and static training. According to the duration, exercise can be divided into short- or long-term exercise, while the frequency of training can also be further subdivided into several groups according to the goal of the study [8]. Exercise must meet different criteria according to the purpose of the research. As exercise research studies are designed for assessing the impact of physical activity on several organ systems, it is crucial to optimize the exercise protocol according to the goal of the study. Furthermore, for the successful outcome, exercise training must consist of several fundamental phases including regeneration time. At the beginning of the study (Phase I), animals should be familiarized with the applied training in order to prevent any injuries or exercise-induced stress. In this phase, adaptation to the environment as well as acquaintance with new forms of movement (e.g., swimming or running) takes place. Then, the planned training with the appropriate intensity, modality, and duration is performed (Phase II). Last but not least, in the case of a daily exercise period, resting time is also necessary for the animals (Phase III) in order to restore physical energy after training [8]. These previously described factors fundamentally determine the outcome of the experiment; thus, their understanding and accurate application are essential for adaptation to human physiology. In the following, we summarize the most often used aerobic and anaerobic training models with their possible areas of application.

\section{Aerobic Exercise Models}

The most commonly used aerobic exercise models in different research fields are voluntary wheel running, forced wheel running, swimming, and treadmill running (Table 1). The aim of these studies can be twofold: to determine the role of sport in disease prevention or to allocate its wideranging effects on preexisting disorders.

4.1. Wheel Running. Voluntary wheel running is a form of exercise where animals have free access to a metal wheel for the whole training time. The running wheel is usually built into the cages of the animals; therefore, they can use the apparatus according to their needs at a lower intensity, any time of the day for any length of time [76]. Running wheels are suitable for smaller rodents (e.g., mice or rats) and are nowadays often equipped with an activity tracking device, which allows the scientist to track down the running distance of the animals. This recreational training is the most stress-free modality of exercise; thus, it is suitable for aging studies and also in conditions where it is important to avoid strenuous exercise [30]. As for the duration of the experiment, voluntary wheel running is applicable for shortand long-term interventions as well. Due to its voluntary, nonstrenuous nature, it is often used for cardiovascular and metabolic studies, but it is suitable for almost every research area [78-80]. Long-term voluntary wheel running is considered to be protective against cardiac injury [81] and an effective tool to enhance antioxidant mechanisms [82]. According to Cunha et al., 3-week-long wheel running improved overall antioxidant status in mice [83]. Additionally, 12 weeks of wheel running exercise exerted its positive influence on lipid metabolism by resulting in a significant decrease in the level of plasma triglyceride and leptin [21]. Along with metabolic effects, long-term voluntary wheel running favorably affected bone properties as well in young mice [84]. Voluntary wheel running was also a convenient exercise protocol in neurodegenerative disorders, as it effectively mitigated impaired spatial memory and neuropathological changes in aging rats through complex biochemical processes [85]. This type of aerobic exercise is also a popular therapeutic approach to tumor prevention and treatment in cancer research [86].

A very similar form of movement to voluntary wheel running is forced wheel running. Forced wheel running differs from the previously mentioned form of wheel running in that its wheel is centrally motorized. This automatically rotating wheel is connected to a specific software program, which allows the scientist to adjust training intensity throughout the running from low to intermediate levels. This training modality offers better control of exercise parameters compared to voluntary wheel running. Forced wheel running is suitable for short-term and long-term interventions as well, depending on the purpose of the study. Similar to voluntary wheel running, this type of exercise is applicable in many areas of research.

4.2. Treadmill Running. Treadmill running is considered to be a forced training model, usually applied with smaller rodents or dogs. Unlike voluntary wheel running, during this exercise, animals are removed from their cages and forced to run on a treadmill. Scientists can change several parameters according to the goal of the study; it allows them to perform moderate- or high-intensity training by adjusting speed, duration, or inclination [76]. Treadmill running is a widely used exercise modality, especially in cardiovascular or metabolic research studies. It has been proved that highintensity treadmill training is an efficient method to reduce cardiovascular risk factors. Haram et al. confirmed that it was able to decrease blood pressure and improve endothelial function and different metabolic parameters as well [87]. It was also reported that high-intensity exercise stimulated mitochondrial biogenesis, thereby contributing to cardiac improvements [88]. Furthermore, this kind of exercise is an applicable method to recreate exercise-induced physiological cardiac hypertrophy. Kemi et al. proved that 4 weeks of intensity-controlled treadmill running caused elevated ventricular weights and normalized the structure and 


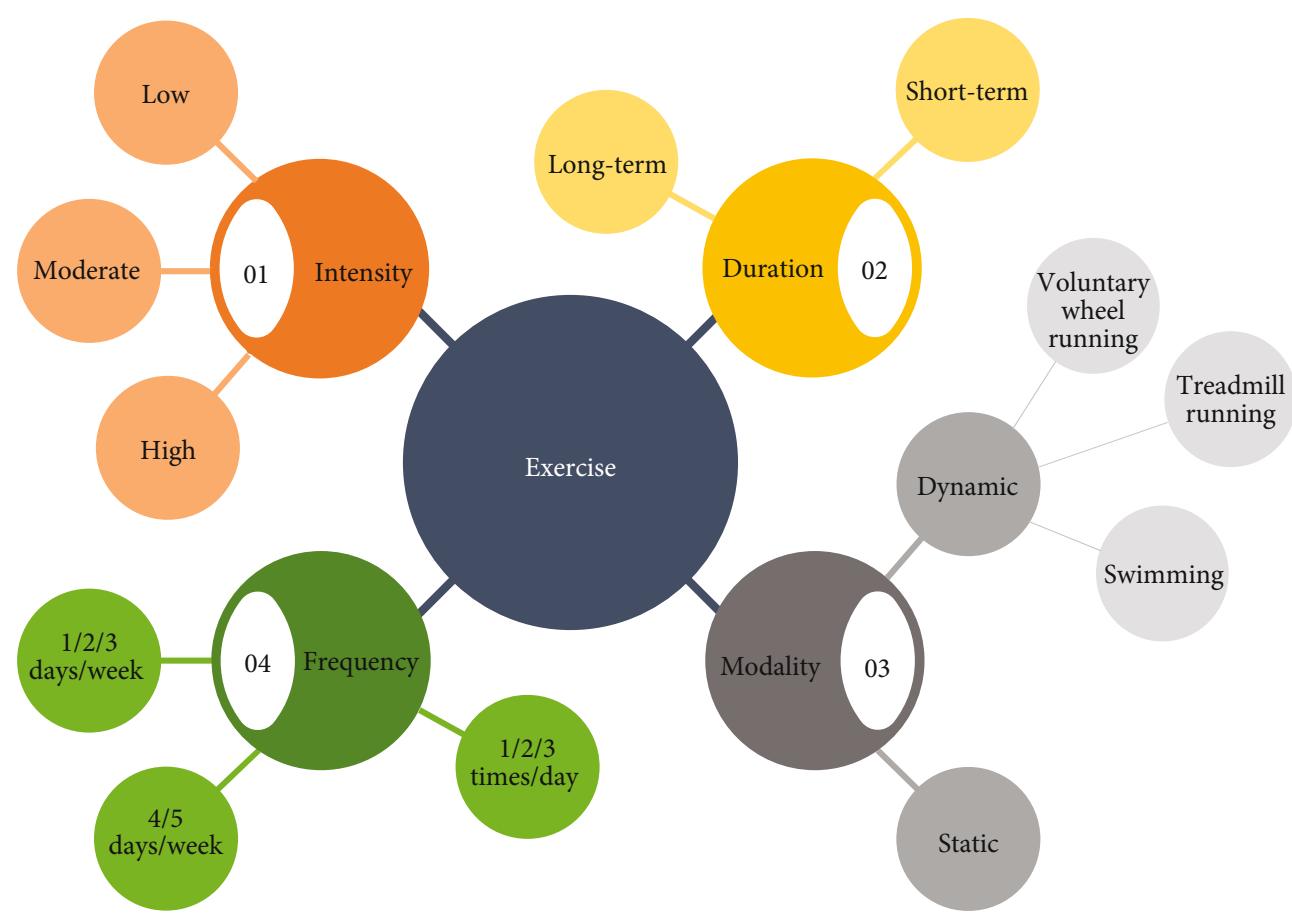

FIGURE 1: Fundamental elements of the exercise protocol. Intensity, duration, frequency, and modality are the four key components of an exercise protocol. Further variants of these subgroups can be used to refine the form of training.

TABle 1: Detailed summary of different exercise modalities (advantages, disadvantages, and areas of application).

\begin{tabular}{|c|c|c|c|}
\hline Type of exercise & Most common research areas & Advantage & Disadvantage \\
\hline \multicolumn{4}{|l|}{ Aerobic } \\
\hline $\begin{array}{l}\text { Voluntary wheel } \\
\text { running }\end{array}$ & $\begin{array}{l}\text { Aging, cardiovascular research, behavioral research, cancer research, } \\
\text { metabolic research, stroke, liver and kidney disease, bone and muscle } \\
\text { physiology, memory }\end{array}$ & Nonstressful & $\begin{array}{l}\text { Uncontrollable } \\
\text { (intensity, } \\
\text { duration) } \\
\text { Possible paw } \\
\text { injuries }\end{array}$ \\
\hline $\begin{array}{l}\text { Forced wheel } \\
\text { running }\end{array}$ & $\begin{array}{l}\text { Aging, cardiovascular research, behavioral research, cancer research, } \\
\text { metabolic research, stroke, liver and kidney disease, bone and muscle } \\
\text { physiology, memory }\end{array}$ & $\begin{array}{l}\text { Controllable (intensity, } \\
\text { duration, frequency) }\end{array}$ & $\begin{array}{l}\text { Stressful } \\
\text { Possible paw } \\
\text { injuries }\end{array}$ \\
\hline $\begin{array}{l}\text { Treadmill } \\
\text { running }\end{array}$ & $\begin{array}{l}\text { Cardiovascular research, behavioral research, cancer, metabolic research, } \\
\text { stroke, liver and kidney disease, bone and muscle physiology, memory }\end{array}$ & $\begin{array}{l}\text { Controllable (intensity, } \\
\text { duration, frequency) }\end{array}$ & $\begin{array}{c}\text { Stressful } \\
\text { Possible paw } \\
\text { injuries } \\
\text { Expensive } \\
\text { apparatus }\end{array}$ \\
\hline Swimming & $\begin{array}{l}\text { Aging, cardiovascular research, behavioral research, cancer, metabolic } \\
\text { research, stroke, liver and kidney disease, bone and muscle physiology, } \\
\text { memory, spinal cord injury }\end{array}$ & $\begin{array}{l}\text { No paw injuries } \\
\text { Less expensive } \\
\text { apparatus }\end{array}$ & Stressful \\
\hline \multicolumn{4}{|c|}{ Anaerobic (resistance) } \\
\hline Ladder climbing & $\begin{array}{l}\text { Memory, behavioral research } \\
\text { Muscle hypertrophy model }\end{array}$ & $\begin{array}{l}\text { With familiarization, it } \\
\text { is less stressful }\end{array}$ & $\begin{array}{l}\text { Long } \\
\text { familiarization } \\
\text { process }\end{array}$ \\
\hline Weight lifting & Muscle hypertrophy model & $\begin{array}{l}\text { Similar to human } \\
\text { training } \\
\text { Quantitative }\end{array}$ & $\begin{array}{l}\text { Stressful to } \\
\text { animals } \\
\text { Special equipment } \\
\text { is needed }\end{array}$ \\
\hline $\begin{array}{l}\text { Electric } \\
\text { stimulation of the } \\
\text { muscles }\end{array}$ & $\begin{array}{l}\text { Muscle hypertrophy model } \\
\text { Muscle injury }\end{array}$ & $\begin{array}{c}\text { Controlled muscle } \\
\text { stimulation } \\
\text { Quantitative }\end{array}$ & $\begin{array}{c}\text { Anesthesia } \\
\text { Surgery } \\
\text { Artificial muscle } \\
\text { contraction }\end{array}$ \\
\hline
\end{tabular}


function of the heart in female and male mice [89]. The work of Kim and Hwang discussed that a short-term (3 weeks) treadmill training was able to improve oxidative parameters in rats with cardiomyopathy [90], while the work of Cechetti et al. demonstrated that a moderate-intensity treadmill training mitigated oxidative damage in the rat hippocampus, therefore contributing to cognitive improvements [91]. According to the results of $\mathrm{Wu}$ et al., 9 weeks of treadmill exercise has beneficial effects against depression-like behavior in rats [92]. In addition, 3 weeks of forced running has also been shown to be beneficial in doxorubicin-induced liver disease through the normalization of oxidative stress markers [93]. Treadmill running is an often-applied exercise modality in cardiovascular therapy-connected research studies; however, conditions occurring during this kind of exercise are generally stressful, which circumstances may interfere with the experimental results, and for that reason, it is not recommended in aging studies. In addition to these areas of use, this type of training at different intensities is able to change the microstructure of the bones, according to Liu et al. [94]. Treadmill running is effective in a hormone-depleted female rat model as well, as its long-term application significantly increased bone mass and strength in young and adult rats [95].

4.3. Swimming. Similar to treadmill running, swimming is also a forced training model. This exercise modality obviously requires a simple swimming apparatus (e.g., a tank), which has to be large enough for the training. It is filled with $30-32^{\circ} \mathrm{C}$ water, the depth of which must be appropriate to the size of the animal [76]. In order to minimize the water-induced stress response, animals must be familiarized with the environment before the experiment. Unlike in the case of running exercises, here, sedentary control animals should also be placed in shallow water in order to exclude the stressful effects of water [96]. In this type of exercise, both the duration and the frequency can be adjusted according to the purpose of the experiment. Based on these factors, we can distinguish between the moderate- and strenuousintensity exercises. Moderate training means 1 hour/day, 5 days/week for 8 weeks, while strenuous exercise requires an increasing duration of the sessions, finally reaching a 2.5hour-long training period/day for also 8 weeks [97]. Furthermore, the swimming procedure can be used as an aerobic exercise with or without an attached weight workload [98]. Swimming with extra weight allows us to study the cardiovascular effects of an exhaustive, strenuous exercise. Olah et al. proved that a 3-hour-long swimming exercise with an extra $5 \%$ body weight attached to the animal resulted in elevated plasma troponin $\mathrm{T}$ and creatine kinase. Furthermore, they demonstrated that this kind of exhaustive training caused elevated apoptotic signaling and matrix metalloprotease dysregulation in the heart [99].

Moderate-intensity swimming, however, is a suitable exercise protocol to study physiological hypertrophy, similar to treadmill running. The findings of Evangelista et al. demonstrated that $90 \mathrm{~min}$ of swimming twice a day, 5 days a week for a 4-week-long period, induced physiological hypertrophy in mice and contributed to normalized heart function [100]. Besides cardiovascular effects, moderateintensity swimming was proved to be efficient in complex metabolic mechanisms. Moustafa and Arisha clarified the beneficial changes of swimming exercise in terms of metabolic alterations [101]. Short-term swimming could be effective by decreasing blood glucose levels and improving insulin-connected pathways in diabetic rats [102]. Besides metabolic influence, swimming exerts anti-inflammatory effects by reducing proinflammatory cytokines in diabetic rats according to de Lemos et al. [103]. It has also been proven that 8 weeks of swimming training successfully mitigated the oxidative damage of the brain and increased its antioxidant status as well [104]. Similarly, Stone et al. found that moderate-intensity swimming was able to upregulate the expression of GSH, SOD, and catalase enzymes, thus ameliorating the antioxidant properties of the hippocampus [105]. According to the latest findings of Alomari et al., short-term swimming resulted in a significant improvement in short- and long-term memories in rats [106]. In this context, Park et al. proved that swimming ameliorated memory defects and psychological disorders by increasing serotonin expression and neurogenesis [107]. The areas of application of swimming extend to the osteoskeletal system as well, as clear results were obtained by Hart et al., who proved that 12-week-long swimming training was effective in the improvement of bone density, structure, and formation in a hormone-depleted female rat model [108].

\section{Resistance (Anaerobic) Training}

Resistance training is an exercise modality designed to enhance muscular strength, power, or physical capacity. In this type of training, external assistance (e.g., electric stimuli, surgery, and specific equipment) is essential to provoke the animals to perform the exercise. Resistance training is usually used for studies in connection with cognitive function and muscle hypertrophy or atrophy $[11,109]$.

5.1. Ladder Climbing. In this type of resistance training, rats are trained to climb a ladder with a load apparatus stabilized to their tail. To perform ladder climbing, no noxious stimuli or motivators are necessarily needed; thus, it can be considered a voluntary exercise. Animals need to be progressively familiarized and trained with climbing the specially designed ladder before the experiment. The intensity of this exercise is defined by how many climbing repetitions are performed during one training phase. It can be applied as a shortterm or long-term exercise model as well. Due to the increased muscle workload, in this type of exercise modality, significant muscle hypertrophy is obtained [110]. Jung et al. clarified that 8 weeks of ladder climbing upregulated the muscle hypertrophy-related myokines in young and adult rats [111]. In addition to muscle hypertrophy, ladder models are increasingly used for studies involving the central nervous system. In this context, according to the results of Cassilhas et al., 8 weeks of ladder climbing exercise improved hippocampus-dependent memory tasks in rats [10]. It also increases cell proliferation and the expression of antiapoptotic proteins in the hippocampus [112]. 
5.2. Weight Lifting. Unlike humans, rodents can perform weight lifting by standing upright and lengthening their hindlimbs. In a specific squat training apparatus, additional weight is added to the animals by using a belt or a shoulder harness. The main disadvantage of this type of resistance training is the use of harmful stimuli in order to motivate the animals to complete the training. This protocol results in nearly $20 \%$ hypertrophy of the leg muscles; thus, it is suitable for research on muscle development [113].

5.3. Electric Stimulation of the Muscles. In order to perform this protocol, animals must be anesthetized. This model also requires an implanted electric stimulator placed into the muscle to be examined [114]. Scientists can control the degree of electric stimulation, which can occur bilaterally or unilaterally. This modality of training evokes significant muscle hypertrophy [115]. The advantage of this protocol is that cooperation of the animals is less needed as a result of anesthesia, although this also implies its disadvantage, as anesthetics may influence the physiology of the animal [76].

\section{Concluding Remarks}

All things considered, the positive effects of physical activity on overall health are unquestionable. Even though animal exercise models have their own limitations, the data obtained through their applications could bring us closer to solving global health issues. As seen, even short-term training can upregulate the antioxidant defense system and induce multifaceted beneficial effects throughout the body. With this nonpharmacological, health-promoting tool, a large percentage of noncommunicable diseases, including metabolic syndrome, CVDs, or even cancer, could be averted. In order to gain a better insight into exercise physiology and its impacts on health status, well-designed animal models are needed.

\section{Conflicts of Interest}

The authors declare no conflict of interest.

\section{Acknowledgments}

This work was supported by GINOP (2.3.2-15-2016-00062), and the Ministry of Human Capacities, Hungary (grant 20391-3/2018/FEKUSTRAT) is acknowledged. Furthermore, this study has also been supported by the New National Excellence Program of the Ministry for Innovation and Technology from the source of the National Research, Development and Innovation Fund ÚNKP-20-4 (to R. Szabó) and ÚNKP-20-3 (to D. Börzsei) and by 2020-1.1.2PIACI-KFI-2020-00154. The study was prepared with the professional support of the Doctoral Student Scholarship Program of the Co-Operative Doctoral Program of the Ministry for Innovation and Technology financed from the National Research, Development and Innovation Fund. Additionally, the Szegedi Tudományegyetem (University of Szeged) Open Access Fund (5525) was granted.

\section{References}

[1] G. N. Ruegsegger and F. W. Booth, "Health benefits of exercise," Cold Spring Harbor Perspectives in Medicine, vol. 8, no. 7, 2018.

[2] C. Cunningham, R. O' Sullivan, P. Caserotti, and M. A. Tully, "Consequences of physical inactivity in older adults: a systematic review of reviews and meta-analyses," Scandinavian Journal of Medicine \& Science in Sports, vol. 30, no. 5, pp. 816-827, 2020.

[3] J. H. Park, J. H. Moon, H. J. Kim, M. H. Kong, and Y. H. Oh, "Sedentary lifestyle: overview of updated evidence of potential health risks," Korean Journal of Family Medicine, vol. 41, no. 6, pp. 365-373, 2020.

[4] F. C. Garton, K. N. North, L. G. Koch, S. L. Britton, G. Nogales-Gadea, and A. Lucia, "Rodent models for resolving extremes of exercise and health," Physiological Genomics, vol. 48, no. 2, pp. 82-92, 2016.

[5] S. Guo, Y. Huang, Y. Zhang, H. Huang, S. Hong, and T. Liu, "Impacts of exercise interventions on different diseases and organ functions in mice," Journal of Sport and Health Science, vol. 9, no. 1, pp. 53-73, 2020.

[6] U. Laufs, S. Wassmann, T. Czech et al., "Physical inactivity increases oxidative stress, endothelial dysfunction, and atherosclerosis," Arteriosclerosis, Thrombosis, and Vascular Biology, vol. 25, no. 4, pp. 809-814, 2005.

[7] V. T. Thu, H. K. Kim, and J. Han, "Acute and chronic exercise in animal models," Advances in Experimental Medicine and Biology, vol. 999, pp. 55-71, 2017.

[8] R. Feng, L. Wang, Z. Li et al., "A systematic comparison of exercise training protocols on animal models of cardiovascular capacity," Life Sciences, vol. 217, pp. 128-140, 2019.

[9] D. Y. Seo, S. R. Lee, N. Kim, K. S. Ko, B. D. Rhee, and J. Han, "Humanized animal exercise model for clinical implication," Pflügers Archiv - European Journal of Physiology, vol. 466, no. 9, pp. 1673-1687, 2014.

[10] R. C. Cassilhas, K. S. Lee, D. P. Venâncio, M. G. Oliveira, S. Tufik, and M. T. de Mello, "Resistance exercise improves hippocampus-dependent memory," Brazilian Journal of Medical and Biological Research, vol. 45, no. 12, pp. 12151220, 2012.

[11] H. Nicastro, N. E. Zanchi, C. R. da Luz, and A. H. Lancha Jr, "Functional and morphological effects of resistance exercise on disuse-induced skeletal muscle atrophy," Brazilian Journal of Medical and Biological Research, vol. 44, no. 11, pp. 1070-1079, 2011.

[12] X. Lin, D. Xiong, Y. Q. Peng et al., "Epidemiology and management of osteoporosis in the People's Republic of China: current perspectives," Clinical Interventions in Aging, vol. 10, pp. 1017-1033, 2015.

[13] X. Chen, L. Li, J. Guo et al., "Treadmill running exercise prevents senile osteoporosis and upregulates the Wnt signaling pathway in SAMP6 mice," Oncotarget, vol. 7, no. 44, pp. 71072-71086, 2016.

[14] L. Zhang, X. Chen, J. Wu et al., "The effects of different intensities of exercise and active vitamin $\mathrm{D}$ on mouse bone mass and bone strength," Journal of Bone and Mineral Metabolism, vol. 35, no. 3, pp. 265-277, 2017.

[15] J. O. Brett, M. Arjona, M. Ikeda et al., "Exercise rejuvenates quiescent skeletal muscle stem cells in old mice through restoration of cyclin D1," Nature Metabolism, vol. 2, no. 4, pp. 307-317, 2020. 
[16] Z. White, J. Terrill, R. B. White et al., "Voluntary resistance wheel exercise from mid-life prevents sarcopenia and increases markers of mitochondrial function and autophagy in muscles of old male and female C57BL/6J mice," Skeletal Muscle, vol. 6, no. 1, p. 45, 2016.

[17] J. E. Donnelly, S. N. Blair, J. M. Jakicic et al., “Appropriate physical activity intervention strategies for weight loss and prevention of weight regain for adults," Medicine and Science in Sports and Exercise, vol. 41, no. 2, pp. 459-471, 2009.

[18] C. A. Slentz, L. A. Bateman, L. H. Willis et al., "Effects of exercise training alone vs a combined exercise and nutritional lifestyle intervention on glucose homeostasis in prediabetic individuals: a randomised controlled trial," Diabetologia, vol. 59, no. 10, pp. 2088-2098, 2016.

[19] V. S. Conn, R. J. Koopman, T. M. Ruppar, L. J. Phillips, D. R. Mehr, and A. R. Hafdahl, "Insulin sensitivity following exercise interventions: systematic review and meta-analysis of outcomes among healthy adults," Journal of Primary Care and Community Health, vol. 5, no. 3, pp. 211-222, 2014.

[20] S. Zanuso, M. Sacchetti, C. J. Sundberg, G. Orlando, P. Benvenuti, and S. Balducci, "Exercise in type 2 diabetes: genetic, metabolic and neuromuscular adaptations. A review of the evidence," British Journal of Sports Medicine, vol. 51, no. 21, pp. 1533-1538, 2017.

[21] A. Posa, R. Szabo, K. Kupai et al., "Exercise training and calorie restriction influence the metabolic parameters in ovariectomized female rats," Oxidative Medicine and Cellular Longevity, vol. 2015, Article ID 787063, 8 pages, 2015.

[22] P. W. Caponi, A. M. Lehnen, G. H. Pinto et al., "Aerobic exercise training induces metabolic benefits in rats with metabolic syndrome independent of dietary changes," Clinics, vol. 68, no. 7, pp. 1010-1017, 2013.

[23] J. Yu, J. Zheng, X. F. Liu et al., "Exercise improved lipid metabolism and insulin sensitivity in rats fed a high-fat diet by regulating glucose transporter 4 (GLUT4) and musclin expression," Brazilian Journal of Medical and Biological Research, vol. 49, no. 5, article e5129, 2016.

[24] R. B. Vega, J. P. Konhilas, D. P. Kelly, and L. A. Leinwand, "Molecular mechanisms underlying cardiac adaptation to exercise," Cell Metabolism, vol. 25, no. 5, pp. 1012-1026, 2017.

[25] X. Lin, X. Zhang, J. Guo et al., "Effects of exercise training on cardiorespiratory fitness and biomarkers of cardiometabolic health: a systematic review and meta-analysis of randomized controlled trials," Journal of the American Heart Association, vol. 4, no. 7, 2015

[26] M. A. Nystoriak and A. Bhatnagar, "Cardiovascular effects and benefits of exercise," Frontiers in Cardiovascular Medicine, vol. 5, p. 135, 2018.

[27] M. E. Davis, H. Cai, L. McCann, T. Fukai, and D. G. Harrison, "Role of c-Src in regulation of endothelial nitric oxide synthase expression during exercise training," American Journal of Physiology Heart and Circulatory Physiology, vol. 284, no. 4, pp. H1449-H1453, 2003.

[28] O. Kuru, Ü. Şentürk, N. Demir, A. Yeşilkaya, G. Ergüler, and M. Erkılıç, "Effect of exercise on blood pressure in rats with chronic NOS inhibition," European Journal of Applied Physiology, vol. 87, no. 2, pp. 134-140, 2002.

[29] J. W. Calvert, M. E. Condit, J. P. Aragon et al., "Exercise protects against myocardial ischemia-reperfusion injury via stimulation of $\beta_{3}$-adrenergic receptors and increased nitric oxide signaling: role of nitrite and nitrosothiols," Circulation Research, vol. 108, no. 12, pp. 1448-1458, 2011.

[30] D. Borzsei, D. Priksz, R. Szabo et al., "Exercise-mitigated sexbased differences in aging: from genetic alterations to heart performance," American Journal of Physiology Heart and Circulatory Physiology, vol. 320, no. 2, pp. H854-H866, 2021.

[31] R. Szabo, D. Borzsei, Z. Karacsonyi et al., "Postconditioninglike effect of exercis: new paradigm in experimental menopause," American Journal of Physiology Heart and Circulatory Physiology, vol. 316, no. 2, pp. H400-H407, 2019.

[32] Y. Xing, S. D. Yang, M. M. Wang, Y. S. Feng, F. Dong, and F. Zhang, "The beneficial role of exercise training for myocardial infarction treatment in elderly," Frontiers in Physiology, vol. 11, p. 270, 2020.

[33] J. Gomes-Osman, D. F. Cabral, C. Hinchman, A. Jannati, T. P. Morris, and A. Pascual-Leone, "The effects of exercise on cognitive function and brain plasticity - a feasibility trial," Restorative Neurology and Neuroscience, vol. 35, no. 5, pp. 547-556, 2017.

[34] J. Gomes-Osman, D. F. Cabral, T. P. Morris et al., "Exercise for cognitive brain health in aging: a systematic review for an evaluation of dose," Neurology: Clinical Practice, vol. 8, no. 3, pp. 257-265, 2018.

[35] J. G. Silveira-Rodrigues, W. Pires, P. F. Gomes et al., "Combined exercise training improves specific domains of cognitive functions and metabolic markers in middle-aged and older adults with type 2 diabetes mellitus," Diabetes Research and Clinical Practice, vol. 173, article 108700, 2021.

[36] R. C. Cassilhas, S. Tufik, and M. T. de Mello, "Physical exercise, neuroplasticity, spatial learning and memory," Cellular and Molecular Life Sciences, vol. 73, no. 5, pp. 975-983, 2016.

[37] D. F. Cabral, J. Rice, T. P. Morris, T. Rundek, A. PascualLeone, and J. Gomes-Osman, "Exercise for brain health: an investigation into the underlying mechanisms guided by dose," Neurotherapeutics, vol. 16, no. 3, pp. 580-599, 2019.

[38] M. M. Kleemeyer, S. Kuhn, J. Prindle et al., "Changes in fitness are associated with changes in hippocampal microstructure and hippocampal volume among older adults," NeuroImage, vol. 131, pp. 155-161, 2016.

[39] R. Ruscheweyh, C. Willemer, K. Kruger et al., "Physical activity and memory functions: an interventional study," Neurobiology of Aging, vol. 32, no. 7, pp. 1304-1319, 2011.

[40] H. van Praag, T. Shubert, C. Zhao, and F. H. Gage, "Exercise enhances learning and hippocampal neurogenesis in aged mice," The Journal of Neuroscience, vol. 25, no. 38, pp. 8680-8685, 2005.

[41] Q. Ding, S. Vaynman, M. Akhavan, Z. Ying, and F. GomezPinilla, "Insulin-like growth factor I interfaces with brainderived neurotrophic factor-mediated synaptic plasticity to modulate aspects of exercise-induced cognitive function," Neuroscience, vol. 140, no. 3, pp. 823-833, 2006.

[42] Y. H. Ding, J. Li, Y. Zhou, J. A. Rafols, J. C. Clark, and Y. Ding, "Cerebral angiogenesis and expression of angiogenic factors in aging rats after exercise," Current Neurovascular Research, vol. 3, no. 1, pp. 15-23, 2006.

[43] Y. Liu, T. Yan, J. M. Chu et al., "The beneficial effects of physical exercise in the brain and related pathophysiological mechanisms in neurodegenerative diseases," Laboratory Investigation, vol. 99, no. 7, pp. 943-957, 2019.

[44] K. M. Moore, R. E. Girens, S. K. Larson et al., "A spectrum of exercise training reduces soluble $\mathrm{A} \beta$ in a dose-dependent 
manner in a mouse model of Alzheimer's disease," Neurobiology of Disease, vol. 85, pp. 218-224, 2016.

[45] J. H. Koo, E. B. Kang, Y. S. Oh, D. S. Yang, and J. Y. Cho, "Treadmill exercise decreases amyloid- $\beta$ burden possibly via activation of SIRT-1 signaling in a mouse model of Alzheimer's disease," Experimental Neurology, vol. 288, pp. 142-152, 2017.

[46] H. Ye, X. Du, and Q. Hua, "Effects of voluntary exercise on antiretroviral therapy-induced neuropathic pain in mice," The Journal of Physiological Sciences, vol. 68, no. 4, pp. 521530, 2018.

[47] T. Tsuchida, Y. A. Lee, N. Fujiwara et al., "A simple diet- and chemical-induced murine NASH model with rapid progression of steatohepatitis, fibrosis and liver cancer," Journal of Hepatology, vol. 69, no. 2, pp. 385-395, 2018.

[48] L. L. Craft and F. M. Perna, "The benefits of exercise for the clinically depressed," Primary Care Companion to the Journal of Clinical Psychiatry, vol. 6, no. 3, pp. 104-111, 2004.

[49] B. K. Pedersen, "Anti-inflammatory effects of exercise: role in diabetes and cardiovascular disease," European Journal of Clinical Investigation, vol. 47, no. 8, pp. 600-611, 2017.

[50] K. M. Beavers, T. E. Brinkley, and B. J. Nicklas, "Effect of exercise training on chronic inflammation," Clinica Chimica Acta, vol. 411, no. 11-12, pp. 785-793, 2010.

[51] C. Kasapis and P. D. Thompson, "The effects of physical activity on serum C-reactive protein and inflammatory markers: a systematic review," Journal of the American College of Cardiology, vol. 45, no. 10, pp. 1563-1569, 2005.

[52] C. Simioni, G. Zauli, A. M. Martelli et al., "Oxidative stress: role of physical exercise and antioxidant nutraceuticals in adulthood and aging," Oncotarget, vol. 9, no. 24, pp. 1718117198, 2018.

[53] M. J. Harutyunyan, A. B. Mathiasen, P. Winkel et al., "Highsensitivity C-reactive protein and N-terminal pro-B-type natriuretic peptide in patients with stable coronary artery disease: a prognostic study within the CLARICOR trial," Scandinavian Journal of Clinical and Laboratory Investigation, vol. 71, no. 1, pp. 52-62, 2011.

[54] M. A. Bouzid, E. Filaire, A. McCall, and C. Fabre, "Radical oxygen species, exercise and aging: an update," Sports Medicine, vol. 45, no. 9, pp. 1245-1261, 2015.

[55] S. Golbidi, M. Badran, and I. Laher, "Antioxidant and antiinflammatory effects of exercise in diabetic patients," Experimental Diabetes Research, vol. 2012, Article ID 941868, 16 pages, 2012.

[56] S. B. Baltaci, R. Mogulkoc, and A. K. Baltaci, "Resveratrol and exercise," Biomedical Reports, vol. 5, no. 5, pp. 525-530, 2016.

[57] J. M. McBride, J. B. Blaak, and T. Triplett-McBride, "Effect of resistance exercise volume and complexity on EMG, strength, and regional body composition," European Journal of Applied Physiology, vol. 90, no. 5-6, pp. 626-632, 2003.

[58] A. S. Elokda and D. H. Nielsen, "Effects of exercise training on the glutathione antioxidant system," European Journal of Cardiovascular Prevention and Rehabilitation, vol. 14, no. 5, pp. 630-637, 2007.

[59] R. Szabo, Z. Karacsonyi, D. Borzsei et al., "Role of exerciseinduced cardiac remodeling in ovariectomized female rats," Oxidative Medicine and Cellular Longevity, vol. 2018, Article ID 6709742, 9 pages, 2018.

[60] Y. Hitomi, S. Watanabe, T. Kizaki et al., "Acute exercise increases expression of extracellular superoxide dismutase in skeletal muscle and the aorta," Redox Report, vol. 13, no. 5, pp. 213-216, 2008.

[61] Z. Yan and H. R. Spaulding, "Extracellular superoxide dismutase, a molecular transducer of health benefits of exercise," Redox Biology, vol. 32, article 101508, 2020.

[62] U. N. Das, "Anti-inflammatory nature of exercise," Nutrition, vol. 20, no. 3, pp. 323-326, 2004.

[63] A. M. Petersen and B. K. Pedersen, "The role of IL-6 in mediating the anti-inflammatory effects of exercise," Journal of Physiology and Pharmacology, vol. 57, Suppl 10, pp. 43-51, 2006.

[64] A. Posa, R. Szabo, A. Csonka et al., "Endogenous estrogenmediated heme oxygenase regulation in experimental menopause," Oxidative Medicine and Cellular Longevity, vol. 2015, Article ID 429713, 7 pages, 2015.

[65] S. C. Moore, I. M. Lee, E. Weiderpass et al., "Association of leisure-time physical activity with risk of 26 types of cancer in 1.44 million adults," JAMA Internal Medicine, vol. 176, no. 6, pp. 816-825, 2016.

[66] K. A. Ashcraft, R. M. Peace, A. S. Betof, M. W. Dewhirst, and L. W. Jones, "Efficacy and mechanisms of aerobic exercise on cancer initiation, progression, and metastasis: a critical systematic review of in vivo preclinical data," Cancer Research, vol. 76, no. 14, pp. 4032-4050, 2016.

[67] L. Pedersen, J. F. Christensen, and P. Hojman, "Effects of exercise on tumor physiology and metabolism," Cancer Journal, vol. 21, no. 2, pp. 111-116, 2015.

[68] A. McTiernan, "Mechanisms linking physical activity with cancer," Nature Reviews Cancer, vol. 8, no. 3, pp. 205-211, 2008.

[69] B. K. Pedersen and L. Hoffman-Goetz, "Exercise and the immune system: regulation, integration, and adaptation," Physiological Reviews, vol. 80, no. 3, pp. 1055-1081, 2000.

[70] M. Catoire and S. Kersten, "The search for exercise factors in humans," The FASEB Journal, vol. 29, no. 5, pp. 1615-1628, 2015.

[71] L. Pedersen, M. Idorn, G. H. Olofsson et al., "Voluntary running suppresses tumor growth through epinephrine- and IL6-dependent NK cell mobilization and redistribution," Cell Metabolism, vol. 23, no. 3, pp. 554-562, 2016.

[72] V. M. Moreira, C. C. da Silva Franco, K. V. Prates et al., "Aerobic exercise training attenuates tumor growth and reduces insulin secretion in Walker 256 tumor-bearing rats," Frontiers in Physiology, vol. 9, p. 465, 2018.

[73] A. Hagar, Z. Wang, S. Koyama et al., "Endurance training slows breast tumor growth in mice by suppressing Treg cells recruitment to tumors," BMC Cancer, vol. 19, no. 1, p. 536, 2019.

[74] C. de Lima, L. Alves, F. Iagher et al., "Tumor growth reduction in Walker 256 tumor-bearing rats performing anaerobic exercise: participation of $\mathrm{Bcl}-2$, Bax, apoptosis, and peroxidation," Applied Physiology, Nutrition, and Metabolism, vol. 36, no. 4, pp. 533-538, 2011.

[75] J. C. Brown, K. Winters-Stone, A. Lee, and K. H. Schmitz, "Cancer, physical activity, and exercise," Comprehensive Physiology, vol. 2, no. 4, pp. 2775-2809, 2012.

[76] J. HJ, "Resource book for the design of animal exercise protocols," American Journal of Veterinary Research, vol. 68, no. 6, p. 583, 2007.

[77] P. B. Laursen, "Training for intense exercise performance: high-intensity or high-volume training?," Scandinavian 
Journal of Medicine \& Science in Sports, vol. 20, Suppl 2, pp. 1-10, 2010.

[78] C. Varga, M. Veszelka, K. Kupai et al., "The effects of exercise training and high triglyceride diet in an estrogen depleted rat model: the role of the heme oxygenase system and inflammatory processes in cardiovascular risk," Journal of Sports Science and Medicine, vol. 17, no. 4, pp. 580-588, 2018.

[79] Y. M. Park, J. Padilla, J. A. Kanaley et al., "Voluntary running attenuates metabolic dysfunction in ovariectomized low-fit rats," Medicine and Science in Sports and Exercise, vol. 49, no. 2, pp. 254-264, 2017.

[80] D. C. Poole, S. W. Copp, T. D. Colburn et al., "Guidelines for animal exercise and training protocols for cardiovascular studies," American Journal of Physiology Heart and Circulatory Physiology, vol. 318, no. 5, pp. H1100-H1138, 2020.

[81] A. Posa, R. Szabo, K. Kupai et al., "Cardioprotective effects of voluntary exercise in a rat model: role of matrix metalloproteinase-2," Oxidative Medicine and Cellular Longevity, vol. 2015, Article ID 876805, 9 pages, 2015.

[82] L. Hoffman-Goetz, N. Pervaiz, and J. Guan, "Voluntary exercise training in mice increases the expression of antioxidant enzymes and decreases the expression of TNF- $\alpha$ in intestinal lymphocytes," Brain, Behavior, and Immunity, vol. 23, no. 4, pp. 498-506, 2009.

[83] M. P. Cunha, D. G. Machado, G. Mancini et al., "The effect of voluntary wheel running on the antioxidant status is dependent on sociability conditions," Pharmacology Biochemistry and Behavior, vol. 198, article 173018, 2020.

[84] H. Ma, S. Torvinen, M. Silvennoinen et al., "Effects of dietinduced obesity and voluntary wheel running on bone properties in young male C57BL/6J mice," Calcified Tissue International, vol. 86, no. 5, pp. 411-419, 2010.

[85] A. Kurucz, M. Bombicz, R. Kiss et al., "Heme oxygenase-1 activity as a correlate to exercise-mediated amelioration of cognitive decline and neuropathological alterations in an aging rat model of dementia," BioMed Research International, vol. 2018, Article ID 7212861, 13 pages, 2018.

[86] X. Zheng, X. X. Cui, M. T. Huang et al., "Inhibitory effect of voluntary running wheel exercise on the growth of human pancreatic Panc-1 and prostate PC-3 xenograft tumors in immunodeficient mice," Oncology Reports, vol. 19, no. 6, pp. 1583-1588, 2008.

[87] P. M. Haram, O. J. Kemi, and U. Wisloff, "Adaptation of endothelium to exercise training: insights from experimental studies," Frontiers in Bioscience, vol. 13, no. 13, pp. 336-346, 2008.

[88] A. D. Hafstad, N. T. Boardman, J. Lund et al., "High intensity interval training alters substrate utilization and reduces oxygen consumption in the heart," Journal of Applied Physiology, vol. 111, no. 5, pp. 1235-1241, 2011.

[89] O. J. Kemi, J. P. Loennechen, U. Wisloff, and O. Ellingsen, "Intensity-controlled treadmill running in mice: cardiac and skeletal muscle hypertrophy," Journal of Applied Physiology, vol. 93, no. 4, pp. 1301-1309, 2002.

[90] E. J. Kim and Hwang, "Effects of aerobic exercise on antioxidants in rat models with cardiomyopathy," Physical Therapy Rehabilitation Science, vol. 4, no. 1, pp. 17-21, 2015.

[91] F. Cechetti, P. V. Worm, V. R. Elsner et al., "Forced treadmill exercise prevents oxidative stress and memory deficits following chronic cerebral hypoperfusion in the rat," Neurobiology of Learning and Memory, vol. 97, no. 1, pp. 90-96, 2012.
[92] T. Wu, Y. Huang, Y. Gong et al., “Treadmill exercise ameliorates depression-like behavior in the rats with prenatal dexamethasone exposure: the role of hippocampal mitochondria," Frontiers in Neuroscience, vol. 13, p. 264, 2019.

[93] M. Ahmadian, V. Dabidi Roshan, and A. S. Leicht, "Agerelated effect of aerobic exercise training on antioxidant and oxidative markers in the liver challenged by doxorubicin in rats," Free Radical Research, vol. 52, no. 7, pp. 775782, 2018.

[94] Z. Liu, J. Gao, and H. Gong, "Effects of treadmill with different intensities on bone quality and muscle properties in adult rats," Biomedical Engineering Online, vol. 18, no. 1, p. 107, 2019.

[95] J. Iwamoto, T. Takeda, and Y. Sato, "Effect of treadmill exercise on bone mass in female rats," Experimental Animals, vol. 54, no. 1, pp. 1-6, 2005.

[96] A. M. Cardoso, F. H. Abdalla, M. D. Bagatini et al., "Swimming training prevents alterations in ecto-NTPDase and adenosine deaminase activities in lymphocytes from $\mathrm{N} \omega$ nitro-L-arginine methyl ester hydrochloride induced hypertension rats," Journal of Hypertension, vol. 33, no. 4, pp. 763-772, 2015.

[97] S. Wang, J. Z. Ma, S. S. Zhu, D. J. Xu, J. G. Zou, and K. J. Cao, "Swimming training can affect intrinsic calcium current characteristics in rat myocardium," European Journal of Applied Physiology, vol. 104, no. 3, pp. 549-555, 2008.

[98] T. W. Oh, F. Ohta, and F. Ohta, "Dose-dependent effect of capsaicin on endurance capacity in rats," The British Journal of Nutrition, vol. 90, no. 3, pp. 515-520, 2003.

[99] A. Olah, B. T. Nemeth, C. Matyas et al., "Cardiac effects of acute exhaustive exercise in a rat model," International Journal of Cardiology, vol. 182, pp. 258-266, 2015.

[100] F. S. Evangelista, P. C. Brum, and J. E. Krieger, "Durationcontrolled swimming exercise training induces cardiac hypertrophy in mice," Brazilian Journal of Medical and Biological Research, vol. 36, no. 12, pp. 1751-1759, 2003.

[101] A. Moustafa and A. H. Arisha, "Swim therapy-induced tissue specific metabolic responses in male rats," Life Sciences, vol. 262, article 118516, 2020.

[102] L. Li, D. He, K. Jiang, and Y. Zhao, "Effects of forced swimming stress on expression and phosphorylation of PI3K/Akt signal pathway in pancreas of type 2 diabetic rats," Ann Transl Med, vol. 8, no. 16, p. 1006, 2020.

[103] E. Teixeira de Lemos, R. Pinto, J. Oliveira et al., "Differential effects of acute (extenuating) and chronic (training) exercise on inflammation and oxidative stress status in an animal model of type 2 diabetes mellitus," Mediators of Inflammation, vol. 2011, Article ID 253061, 8 pages, 2011.

[104] L. F. Nonato, E. Rocha-Vieira, R. Tossige-Gomes et al., "Swimming training attenuates oxidative damage and increases enzymatic but not non-enzymatic antioxidant defenses in the rat brain," Brazilian Journal of Medical and Biological Research, vol. 49, no. 10, article e5310, 2016.

[105] V. Stone, K. Y. Kudo, T. B. Marcelino, P. M. August, and C. Matte, "Swimming exercise enhances the hippocampal antioxidant status of female Wistar rats," Redox Report, vol. 20, no. 3, pp. 133-138, 2015.

[106] M. A. Alomari, K. H. Alzoubi, and O. F. Khabour, "Swimming exercise improves short- and long-term memories: time-course changes," Physiological Reports, vol. 9, no. 11, article e14851, 2021. 
[107] H. S. Park, T. W. Kim, S. S. Park, and S. J. Lee, "Swimming exercise ameliorates mood disorder and memory impairment by enhancing neurogenesis, serotonin expression, and inhibiting apoptosis in social isolation rats during adolescence," Journal of Exercise Rehabilitation, vol. 16, no. 2, pp. 132140, 2020.

[108] K. J. Hart, J. M. Shaw, E. Vajda, M. Hegsted, and S. C. Miller, "Swim-trained rats have greater bone mass, density, strength, and dynamics," Journal of Applied Physiology, vol. 91, no. 4, pp. 1663-1668, 2001.

[109] R. M. Arida, F. A. Scorza, S. G. da Silva, R. M. Cysneiros, and E. A. Cavalheiro, "Exercise paradigms to study brain injury recovery in rodents," American Journal of Physical Medicine \& Rehabilitation, vol. 90, no. 6, pp. 452-465, 2011.

[110] R. Mounier, H. Cavalie, G. Lac, and E. Clottes, "Molecular impact of clenbuterol and isometric strength training on rat EDL muscles," Pflügers Archiv-European Journal of Physiology, vol. 453, no. 4, pp. 497-507, 2007.

[111] S. Jung, N. Ahn, S. Kim et al., "The effect of ladder-climbing exercise on atrophy/hypertrophy-related myokine expression in middle-aged male Wistar rats," The Journal of Physiological Sciences, vol. 65, no. 6, pp. 515-521, 2015.

[112] M. S. Nokia, S. Lensu, J. P. Ahtiainen et al., "Physical exercise increases adult hippocampal neurogenesis in male rats provided it is aerobic and sustained," The Journal of Physiology, vol. 594, no. 7, pp. 1855-1873, 2016.

[113] D. A. Lowe and S. E. Alway, "Animal models for inducing muscle hypertrophy: are they relevant for clinical applications in humans?," The Journal of Orthopaedic and Sports Physical Therapy, vol. 32, no. 2, pp. 36-43, 2002.

[114] Y. Song, S. Forsgren, J. Yu, R. Lorentzon, and P. S. Stal, "Effects on contralateral muscles after unilateral electrical muscle stimulation and exercise," PLoS One, vol. 7, no. 12, article e52230, 2012.

[115] K. Baar and K. Esser, "Phosphorylation of p70S6kcorrelates with increased skeletal muscle mass following resistance exercise," The American Journal of Physiology, vol. 276, no. 1, pp. C120-C127, 1999. 